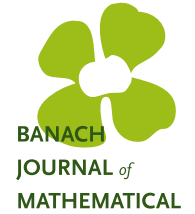

ANALYSIS
Banach J. Math. Anal. 10 (2016), no. 3, 466-481

http://dx.doi.org/10.1215/17358787-3599675

ISSN: $1735-8787$ (electronic)

http://projecteuclid.org/bjma

\title{
CHARACTERIZATIONS OF JORDAN LEFT DERIVATIONS ON SOME ALGEBRAS
}

\author{
GUANGYU AN, YANA DING, and JIANKUI LI* \\ Communicated by A. R. Villena
}

\begin{abstract}
A linear mapping $\delta$ from an algebra $\mathcal{A}$ into a left $\mathcal{A}$-module $\mathcal{M}$ is called a Jordan left derivation if $\delta\left(A^{2}\right)=2 A \delta(A)$ for every $A \in \mathcal{A}$. We prove that if an algebra $\mathcal{A}$ and a left $\mathcal{A}$-module $\mathcal{M}$ satisfy one of the following conditions-(1) $\mathcal{A}$ is a $C^{*}$-algebra and $\mathcal{M}$ is a Banach left $\mathcal{A}$-module; (2) $\mathcal{A}=$ $\operatorname{Alg} \mathcal{L}$ with $\cap\left\{L_{-}: L \in \mathcal{J}_{\mathcal{L}}\right\}=(0)$ and $\mathcal{M}=B(X)$; and (3) $\mathcal{A}$ is a commutative subspace lattice algebra of a von Neumann algebra $\mathcal{B}$ and $\mathcal{M}=B(\mathcal{H})$ - then every Jordan left derivation from $\mathcal{A}$ into $\mathcal{M}$ is zero. $\delta$ is called left derivable at $G \in \mathcal{A}$ if $\delta(A B)=A \delta(B)+B \delta(A)$ for each $A, B \in \mathcal{A}$ with $A B=G$. We show that if $\mathcal{A}$ is a factor von Neumann algebra, $G$ is a left separating point of $\mathcal{A}$ or a nonzero self-adjoint element in $\mathcal{A}$, and $\delta$ is left derivable at $G$, then $\delta \equiv 0$.
\end{abstract}

\section{INTRODUCTION}

Let $\mathcal{R}$ be an associative ring. For an integer $n \geqslant 2, \mathcal{R}$ is said to be $n$-torsion-free if $n A=0$ implies $A=0$ for every $A$ in $\mathcal{R}$. Recall that a ring $\mathcal{R}$ is prime if $A \mathcal{R} B=(0)$ implies that either $A=0$ or $B=0$ for each $A, B$ in $\mathcal{R}$, and it is semiprime if $A \mathcal{R} A=(0)$ implies $A=0$ for every $A$ in $\mathcal{R}$.

Suppose that $\mathcal{M}$ is an $\mathcal{R}$-bimodule. An additive mapping $\delta$ from $\mathcal{R}$ into $\mathcal{M}$ is called a derivation if $\delta(A B)=\delta(A) B+A \delta(B)$ for each $A, B$ in $\mathcal{R}$, and $\delta$ is called a Jordan derivation if $\delta\left(A^{2}\right)=\delta(A) A+A \delta(A)$ for every $A$ in $\mathcal{R}$. Obviously, every derivation is a Jordan derivation. The converse is, in general, not true. A classi-

Copyright 2016 by the Tusi Mathematical Research Group.

Received Apr. 1, 2015; Accepted Aug. 18, 2015.

${ }^{*}$ Corresponding author.

2010 Mathematics Subject Classification. Primary 47B47; Secondary 47L35, 47C15.

Keywords. $C^{*}$-algebra, Jordan left derivation, left derivable point, left separating point. 


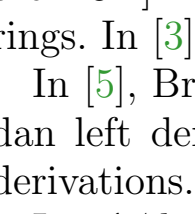

ANALYSIS
Banach J. Math. Anal. 10 (2016), no. 3, 466-481

http://dx.doi.org/10.1215/17358787-3599675

ISSN: $1735-8787$ (electronic)

http://projecteuclid.org/bjma

\title{
CHARACTERIZATIONS OF JORDAN LEFT DERIVATIONS ON SOME ALGEBRAS
}

\author{
GUANGYU AN, YANA DING, and JIANKUI LI* \\ Communicated by A. R. Villena
}

\begin{abstract}
A linear mapping $\delta$ from an algebra $\mathcal{A}$ into a left $\mathcal{A}$-module $\mathcal{M}$ is called a Jordan left derivation if $\delta\left(A^{2}\right)=2 A \delta(A)$ for every $A \in \mathcal{A}$. We prove that if an algebra $\mathcal{A}$ and a left $\mathcal{A}$-module $\mathcal{M}$ satisfy one of the following conditions-(1) $\mathcal{A}$ is a $C^{*}$-algebra and $\mathcal{M}$ is a Banach left $\mathcal{A}$-module; (2) $\mathcal{A}=$ $\operatorname{Alg} \mathcal{L}$ with $\cap\left\{L_{-}: L \in \mathcal{J}_{\mathcal{L}}\right\}=(0)$ and $\mathcal{M}=B(X)$; and (3) $\mathcal{A}$ is a commutative subspace lattice algebra of a von Neumann algebra $\mathcal{B}$ and $\mathcal{M}=B(\mathcal{H})$ - then every Jordan left derivation from $\mathcal{A}$ into $\mathcal{M}$ is zero. $\delta$ is called left derivable at $G \in \mathcal{A}$ if $\delta(A B)=A \delta(B)+B \delta(A)$ for each $A, B \in \mathcal{A}$ with $A B=G$. We show that if $\mathcal{A}$ is a factor von Neumann algebra, $G$ is a left separating point of $\mathcal{A}$ or a nonzero self-adjoint element in $\mathcal{A}$, and $\delta$ is left derivable at $G$, then $\delta \equiv 0$.
\end{abstract}

\section{INTRODUCTION}

Let $\mathcal{R}$ be an associative ring. For an integer $n \geqslant 2, \mathcal{R}$ is said to be $n$-torsion-free if $n A=0$ implies $A=0$ for every $A$ in $\mathcal{R}$. Recall that a ring $\mathcal{R}$ is prime if $A \mathcal{R} B=(0)$ implies that either $A=0$ or $B=0$ for each $A, B$ in $\mathcal{R}$, and it is semiprime if $A \mathcal{R} A=(0)$ implies $A=0$ for every $A$ in $\mathcal{R}$.

Suppose that $\mathcal{M}$ is an $\mathcal{R}$-bimodule. An additive mapping $\delta$ from $\mathcal{R}$ into $\mathcal{M}$ is called a derivation if $\delta(A B)=\delta(A) B+A \delta(B)$ for each $A, B$ in $\mathcal{R}$, and $\delta$ is called a Jordan derivation if $\delta\left(A^{2}\right)=\delta(A) A+A \delta(A)$ for every $A$ in $\mathcal{R}$. Obviously, every derivation is a Jordan derivation. The converse is, in general, not true. A classi-

Copyright 2016 by the Tusi Mathematical Research Group.

Received Apr. 1, 2015; Accepted Aug. 18, 2015.

${ }^{*}$ Corresponding author.

2010 Mathematics Subject Classification. Primary 47B47; Secondary 47L35, 47C15.

Keywords. $C^{*}$-algebra, Jordan left derivation, left derivable point, left separating point. 


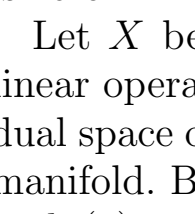

ANALYSIS
Banach J. Math. Anal. 10 (2016), no. 3, 466-481

http://dx.doi.org/10.1215/17358787-3599675

ISSN: $1735-8787$ (electronic)

http://projecteuclid.org/bjma

\title{
CHARACTERIZATIONS OF JORDAN LEFT DERIVATIONS ON SOME ALGEBRAS
}

\author{
GUANGYU AN, YANA DING, and JIANKUI LI* \\ Communicated by A. R. Villena
}

\begin{abstract}
A linear mapping $\delta$ from an algebra $\mathcal{A}$ into a left $\mathcal{A}$-module $\mathcal{M}$ is called a Jordan left derivation if $\delta\left(A^{2}\right)=2 A \delta(A)$ for every $A \in \mathcal{A}$. We prove that if an algebra $\mathcal{A}$ and a left $\mathcal{A}$-module $\mathcal{M}$ satisfy one of the following conditions-(1) $\mathcal{A}$ is a $C^{*}$-algebra and $\mathcal{M}$ is a Banach left $\mathcal{A}$-module; (2) $\mathcal{A}=$ $\operatorname{Alg} \mathcal{L}$ with $\cap\left\{L_{-}: L \in \mathcal{J}_{\mathcal{L}}\right\}=(0)$ and $\mathcal{M}=B(X)$; and (3) $\mathcal{A}$ is a commutative subspace lattice algebra of a von Neumann algebra $\mathcal{B}$ and $\mathcal{M}=B(\mathcal{H})$ - then every Jordan left derivation from $\mathcal{A}$ into $\mathcal{M}$ is zero. $\delta$ is called left derivable at $G \in \mathcal{A}$ if $\delta(A B)=A \delta(B)+B \delta(A)$ for each $A, B \in \mathcal{A}$ with $A B=G$. We show that if $\mathcal{A}$ is a factor von Neumann algebra, $G$ is a left separating point of $\mathcal{A}$ or a nonzero self-adjoint element in $\mathcal{A}$, and $\delta$ is left derivable at $G$, then $\delta \equiv 0$.
\end{abstract}

\section{INTRODUCTION}

Let $\mathcal{R}$ be an associative ring. For an integer $n \geqslant 2, \mathcal{R}$ is said to be $n$-torsion-free if $n A=0$ implies $A=0$ for every $A$ in $\mathcal{R}$. Recall that a ring $\mathcal{R}$ is prime if $A \mathcal{R} B=(0)$ implies that either $A=0$ or $B=0$ for each $A, B$ in $\mathcal{R}$, and it is semiprime if $A \mathcal{R} A=(0)$ implies $A=0$ for every $A$ in $\mathcal{R}$.

Suppose that $\mathcal{M}$ is an $\mathcal{R}$-bimodule. An additive mapping $\delta$ from $\mathcal{R}$ into $\mathcal{M}$ is called a derivation if $\delta(A B)=\delta(A) B+A \delta(B)$ for each $A, B$ in $\mathcal{R}$, and $\delta$ is called a Jordan derivation if $\delta\left(A^{2}\right)=\delta(A) A+A \delta(A)$ for every $A$ in $\mathcal{R}$. Obviously, every derivation is a Jordan derivation. The converse is, in general, not true. A classi-

Copyright 2016 by the Tusi Mathematical Research Group.

Received Apr. 1, 2015; Accepted Aug. 18, 2015.

${ }^{*}$ Corresponding author.

2010 Mathematics Subject Classification. Primary 47B47; Secondary 47L35, 47C15.

Keywords. $C^{*}$-algebra, Jordan left derivation, left derivable point, left separating point. 


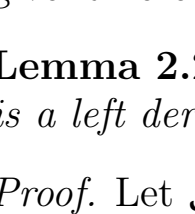

ANALYSIS
Banach J. Math. Anal. 10 (2016), no. 3, 466-481

http://dx.doi.org/10.1215/17358787-3599675

ISSN: $1735-8787$ (electronic)

http://projecteuclid.org/bjma

\title{
CHARACTERIZATIONS OF JORDAN LEFT DERIVATIONS ON SOME ALGEBRAS
}

\author{
GUANGYU AN, YANA DING, and JIANKUI LI* \\ Communicated by A. R. Villena
}

\begin{abstract}
A linear mapping $\delta$ from an algebra $\mathcal{A}$ into a left $\mathcal{A}$-module $\mathcal{M}$ is called a Jordan left derivation if $\delta\left(A^{2}\right)=2 A \delta(A)$ for every $A \in \mathcal{A}$. We prove that if an algebra $\mathcal{A}$ and a left $\mathcal{A}$-module $\mathcal{M}$ satisfy one of the following conditions-(1) $\mathcal{A}$ is a $C^{*}$-algebra and $\mathcal{M}$ is a Banach left $\mathcal{A}$-module; (2) $\mathcal{A}=$ $\operatorname{Alg} \mathcal{L}$ with $\cap\left\{L_{-}: L \in \mathcal{J}_{\mathcal{L}}\right\}=(0)$ and $\mathcal{M}=B(X)$; and (3) $\mathcal{A}$ is a commutative subspace lattice algebra of a von Neumann algebra $\mathcal{B}$ and $\mathcal{M}=B(\mathcal{H})$ - then every Jordan left derivation from $\mathcal{A}$ into $\mathcal{M}$ is zero. $\delta$ is called left derivable at $G \in \mathcal{A}$ if $\delta(A B)=A \delta(B)+B \delta(A)$ for each $A, B \in \mathcal{A}$ with $A B=G$. We show that if $\mathcal{A}$ is a factor von Neumann algebra, $G$ is a left separating point of $\mathcal{A}$ or a nonzero self-adjoint element in $\mathcal{A}$, and $\delta$ is left derivable at $G$, then $\delta \equiv 0$.
\end{abstract}

\section{INTRODUCTION}

Let $\mathcal{R}$ be an associative ring. For an integer $n \geqslant 2, \mathcal{R}$ is said to be $n$-torsion-free if $n A=0$ implies $A=0$ for every $A$ in $\mathcal{R}$. Recall that a ring $\mathcal{R}$ is prime if $A \mathcal{R} B=(0)$ implies that either $A=0$ or $B=0$ for each $A, B$ in $\mathcal{R}$, and it is semiprime if $A \mathcal{R} A=(0)$ implies $A=0$ for every $A$ in $\mathcal{R}$.

Suppose that $\mathcal{M}$ is an $\mathcal{R}$-bimodule. An additive mapping $\delta$ from $\mathcal{R}$ into $\mathcal{M}$ is called a derivation if $\delta(A B)=\delta(A) B+A \delta(B)$ for each $A, B$ in $\mathcal{R}$, and $\delta$ is called a Jordan derivation if $\delta\left(A^{2}\right)=\delta(A) A+A \delta(A)$ for every $A$ in $\mathcal{R}$. Obviously, every derivation is a Jordan derivation. The converse is, in general, not true. A classi-

Copyright 2016 by the Tusi Mathematical Research Group.

Received Apr. 1, 2015; Accepted Aug. 18, 2015.

${ }^{*}$ Corresponding author.

2010 Mathematics Subject Classification. Primary 47B47; Secondary 47L35, 47C15.

Keywords. $C^{*}$-algebra, Jordan left derivation, left derivable point, left separating point. 


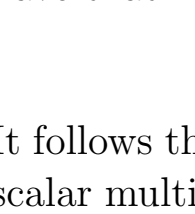

ANALYSIS
Banach J. Math. Anal. 10 (2016), no. 3, 466-481

http://dx.doi.org/10.1215/17358787-3599675

ISSN: $1735-8787$ (electronic)

http://projecteuclid.org/bjma

\title{
CHARACTERIZATIONS OF JORDAN LEFT DERIVATIONS ON SOME ALGEBRAS
}

\author{
GUANGYU AN, YANA DING, and JIANKUI LI* \\ Communicated by A. R. Villena
}

\begin{abstract}
A linear mapping $\delta$ from an algebra $\mathcal{A}$ into a left $\mathcal{A}$-module $\mathcal{M}$ is called a Jordan left derivation if $\delta\left(A^{2}\right)=2 A \delta(A)$ for every $A \in \mathcal{A}$. We prove that if an algebra $\mathcal{A}$ and a left $\mathcal{A}$-module $\mathcal{M}$ satisfy one of the following conditions-(1) $\mathcal{A}$ is a $C^{*}$-algebra and $\mathcal{M}$ is a Banach left $\mathcal{A}$-module; (2) $\mathcal{A}=$ $\operatorname{Alg} \mathcal{L}$ with $\cap\left\{L_{-}: L \in \mathcal{J}_{\mathcal{L}}\right\}=(0)$ and $\mathcal{M}=B(X)$; and (3) $\mathcal{A}$ is a commutative subspace lattice algebra of a von Neumann algebra $\mathcal{B}$ and $\mathcal{M}=B(\mathcal{H})$ - then every Jordan left derivation from $\mathcal{A}$ into $\mathcal{M}$ is zero. $\delta$ is called left derivable at $G \in \mathcal{A}$ if $\delta(A B)=A \delta(B)+B \delta(A)$ for each $A, B \in \mathcal{A}$ with $A B=G$. We show that if $\mathcal{A}$ is a factor von Neumann algebra, $G$ is a left separating point of $\mathcal{A}$ or a nonzero self-adjoint element in $\mathcal{A}$, and $\delta$ is left derivable at $G$, then $\delta \equiv 0$.
\end{abstract}

\section{INTRODUCTION}

Let $\mathcal{R}$ be an associative ring. For an integer $n \geqslant 2, \mathcal{R}$ is said to be $n$-torsion-free if $n A=0$ implies $A=0$ for every $A$ in $\mathcal{R}$. Recall that a ring $\mathcal{R}$ is prime if $A \mathcal{R} B=(0)$ implies that either $A=0$ or $B=0$ for each $A, B$ in $\mathcal{R}$, and it is semiprime if $A \mathcal{R} A=(0)$ implies $A=0$ for every $A$ in $\mathcal{R}$.

Suppose that $\mathcal{M}$ is an $\mathcal{R}$-bimodule. An additive mapping $\delta$ from $\mathcal{R}$ into $\mathcal{M}$ is called a derivation if $\delta(A B)=\delta(A) B+A \delta(B)$ for each $A, B$ in $\mathcal{R}$, and $\delta$ is called a Jordan derivation if $\delta\left(A^{2}\right)=\delta(A) A+A \delta(A)$ for every $A$ in $\mathcal{R}$. Obviously, every derivation is a Jordan derivation. The converse is, in general, not true. A classi-

Copyright 2016 by the Tusi Mathematical Research Group.

Received Apr. 1, 2015; Accepted Aug. 18, 2015.

${ }^{*}$ Corresponding author.

2010 Mathematics Subject Classification. Primary 47B47; Secondary 47L35, 47C15.

Keywords. $C^{*}$-algebra, Jordan left derivation, left derivable point, left separating point. 


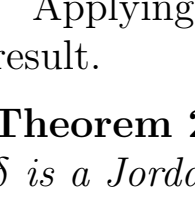

ANALYSIS
Banach J. Math. Anal. 10 (2016), no. 3, 466-481

http://dx.doi.org/10.1215/17358787-3599675

ISSN: $1735-8787$ (electronic)

http://projecteuclid.org/bjma

\title{
CHARACTERIZATIONS OF JORDAN LEFT DERIVATIONS ON SOME ALGEBRAS
}

\author{
GUANGYU AN, YANA DING, and JIANKUI LI* \\ Communicated by A. R. Villena
}

\begin{abstract}
A linear mapping $\delta$ from an algebra $\mathcal{A}$ into a left $\mathcal{A}$-module $\mathcal{M}$ is called a Jordan left derivation if $\delta\left(A^{2}\right)=2 A \delta(A)$ for every $A \in \mathcal{A}$. We prove that if an algebra $\mathcal{A}$ and a left $\mathcal{A}$-module $\mathcal{M}$ satisfy one of the following conditions-(1) $\mathcal{A}$ is a $C^{*}$-algebra and $\mathcal{M}$ is a Banach left $\mathcal{A}$-module; (2) $\mathcal{A}=$ $\operatorname{Alg} \mathcal{L}$ with $\cap\left\{L_{-}: L \in \mathcal{J}_{\mathcal{L}}\right\}=(0)$ and $\mathcal{M}=B(X)$; and (3) $\mathcal{A}$ is a commutative subspace lattice algebra of a von Neumann algebra $\mathcal{B}$ and $\mathcal{M}=B(\mathcal{H})$ - then every Jordan left derivation from $\mathcal{A}$ into $\mathcal{M}$ is zero. $\delta$ is called left derivable at $G \in \mathcal{A}$ if $\delta(A B)=A \delta(B)+B \delta(A)$ for each $A, B \in \mathcal{A}$ with $A B=G$. We show that if $\mathcal{A}$ is a factor von Neumann algebra, $G$ is a left separating point of $\mathcal{A}$ or a nonzero self-adjoint element in $\mathcal{A}$, and $\delta$ is left derivable at $G$, then $\delta \equiv 0$.
\end{abstract}

\section{INTRODUCTION}

Let $\mathcal{R}$ be an associative ring. For an integer $n \geqslant 2, \mathcal{R}$ is said to be $n$-torsion-free if $n A=0$ implies $A=0$ for every $A$ in $\mathcal{R}$. Recall that a ring $\mathcal{R}$ is prime if $A \mathcal{R} B=(0)$ implies that either $A=0$ or $B=0$ for each $A, B$ in $\mathcal{R}$, and it is semiprime if $A \mathcal{R} A=(0)$ implies $A=0$ for every $A$ in $\mathcal{R}$.

Suppose that $\mathcal{M}$ is an $\mathcal{R}$-bimodule. An additive mapping $\delta$ from $\mathcal{R}$ into $\mathcal{M}$ is called a derivation if $\delta(A B)=\delta(A) B+A \delta(B)$ for each $A, B$ in $\mathcal{R}$, and $\delta$ is called a Jordan derivation if $\delta\left(A^{2}\right)=\delta(A) A+A \delta(A)$ for every $A$ in $\mathcal{R}$. Obviously, every derivation is a Jordan derivation. The converse is, in general, not true. A classi-

Copyright 2016 by the Tusi Mathematical Research Group.

Received Apr. 1, 2015; Accepted Aug. 18, 2015.

${ }^{*}$ Corresponding author.

2010 Mathematics Subject Classification. Primary 47B47; Secondary 47L35, 47C15.

Keywords. $C^{*}$-algebra, Jordan left derivation, left derivable point, left separating point. 


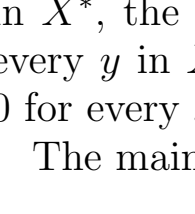

ANALYSIS
Banach J. Math. Anal. 10 (2016), no. 3, 466-481

http://dx.doi.org/10.1215/17358787-3599675

ISSN: $1735-8787$ (electronic)

http://projecteuclid.org/bjma

\title{
CHARACTERIZATIONS OF JORDAN LEFT DERIVATIONS ON SOME ALGEBRAS
}

\author{
GUANGYU AN, YANA DING, and JIANKUI LI* \\ Communicated by A. R. Villena
}

\begin{abstract}
A linear mapping $\delta$ from an algebra $\mathcal{A}$ into a left $\mathcal{A}$-module $\mathcal{M}$ is called a Jordan left derivation if $\delta\left(A^{2}\right)=2 A \delta(A)$ for every $A \in \mathcal{A}$. We prove that if an algebra $\mathcal{A}$ and a left $\mathcal{A}$-module $\mathcal{M}$ satisfy one of the following conditions-(1) $\mathcal{A}$ is a $C^{*}$-algebra and $\mathcal{M}$ is a Banach left $\mathcal{A}$-module; (2) $\mathcal{A}=$ $\operatorname{Alg} \mathcal{L}$ with $\cap\left\{L_{-}: L \in \mathcal{J}_{\mathcal{L}}\right\}=(0)$ and $\mathcal{M}=B(X)$; and (3) $\mathcal{A}$ is a commutative subspace lattice algebra of a von Neumann algebra $\mathcal{B}$ and $\mathcal{M}=B(\mathcal{H})$ - then every Jordan left derivation from $\mathcal{A}$ into $\mathcal{M}$ is zero. $\delta$ is called left derivable at $G \in \mathcal{A}$ if $\delta(A B)=A \delta(B)+B \delta(A)$ for each $A, B \in \mathcal{A}$ with $A B=G$. We show that if $\mathcal{A}$ is a factor von Neumann algebra, $G$ is a left separating point of $\mathcal{A}$ or a nonzero self-adjoint element in $\mathcal{A}$, and $\delta$ is left derivable at $G$, then $\delta \equiv 0$.
\end{abstract}

\section{INTRODUCTION}

Let $\mathcal{R}$ be an associative ring. For an integer $n \geqslant 2, \mathcal{R}$ is said to be $n$-torsion-free if $n A=0$ implies $A=0$ for every $A$ in $\mathcal{R}$. Recall that a ring $\mathcal{R}$ is prime if $A \mathcal{R} B=(0)$ implies that either $A=0$ or $B=0$ for each $A, B$ in $\mathcal{R}$, and it is semiprime if $A \mathcal{R} A=(0)$ implies $A=0$ for every $A$ in $\mathcal{R}$.

Suppose that $\mathcal{M}$ is an $\mathcal{R}$-bimodule. An additive mapping $\delta$ from $\mathcal{R}$ into $\mathcal{M}$ is called a derivation if $\delta(A B)=\delta(A) B+A \delta(B)$ for each $A, B$ in $\mathcal{R}$, and $\delta$ is called a Jordan derivation if $\delta\left(A^{2}\right)=\delta(A) A+A \delta(A)$ for every $A$ in $\mathcal{R}$. Obviously, every derivation is a Jordan derivation. The converse is, in general, not true. A classi-

Copyright 2016 by the Tusi Mathematical Research Group.

Received Apr. 1, 2015; Accepted Aug. 18, 2015.

${ }^{*}$ Corresponding author.

2010 Mathematics Subject Classification. Primary 47B47; Secondary 47L35, 47C15.

Keywords. $C^{*}$-algebra, Jordan left derivation, left derivable point, left separating point. 


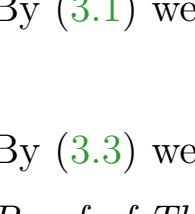

ANALYSIS
Banach J. Math. Anal. 10 (2016), no. 3, 466-481

http://dx.doi.org/10.1215/17358787-3599675

ISSN: $1735-8787$ (electronic)

http://projecteuclid.org/bjma

\title{
CHARACTERIZATIONS OF JORDAN LEFT DERIVATIONS ON SOME ALGEBRAS
}

\author{
GUANGYU AN, YANA DING, and JIANKUI LI* \\ Communicated by A. R. Villena
}

\begin{abstract}
A linear mapping $\delta$ from an algebra $\mathcal{A}$ into a left $\mathcal{A}$-module $\mathcal{M}$ is called a Jordan left derivation if $\delta\left(A^{2}\right)=2 A \delta(A)$ for every $A \in \mathcal{A}$. We prove that if an algebra $\mathcal{A}$ and a left $\mathcal{A}$-module $\mathcal{M}$ satisfy one of the following conditions-(1) $\mathcal{A}$ is a $C^{*}$-algebra and $\mathcal{M}$ is a Banach left $\mathcal{A}$-module; (2) $\mathcal{A}=$ $\operatorname{Alg} \mathcal{L}$ with $\cap\left\{L_{-}: L \in \mathcal{J}_{\mathcal{L}}\right\}=(0)$ and $\mathcal{M}=B(X)$; and (3) $\mathcal{A}$ is a commutative subspace lattice algebra of a von Neumann algebra $\mathcal{B}$ and $\mathcal{M}=B(\mathcal{H})$ - then every Jordan left derivation from $\mathcal{A}$ into $\mathcal{M}$ is zero. $\delta$ is called left derivable at $G \in \mathcal{A}$ if $\delta(A B)=A \delta(B)+B \delta(A)$ for each $A, B \in \mathcal{A}$ with $A B=G$. We show that if $\mathcal{A}$ is a factor von Neumann algebra, $G$ is a left separating point of $\mathcal{A}$ or a nonzero self-adjoint element in $\mathcal{A}$, and $\delta$ is left derivable at $G$, then $\delta \equiv 0$.
\end{abstract}

\section{INTRODUCTION}

Let $\mathcal{R}$ be an associative ring. For an integer $n \geqslant 2, \mathcal{R}$ is said to be $n$-torsion-free if $n A=0$ implies $A=0$ for every $A$ in $\mathcal{R}$. Recall that a ring $\mathcal{R}$ is prime if $A \mathcal{R} B=(0)$ implies that either $A=0$ or $B=0$ for each $A, B$ in $\mathcal{R}$, and it is semiprime if $A \mathcal{R} A=(0)$ implies $A=0$ for every $A$ in $\mathcal{R}$.

Suppose that $\mathcal{M}$ is an $\mathcal{R}$-bimodule. An additive mapping $\delta$ from $\mathcal{R}$ into $\mathcal{M}$ is called a derivation if $\delta(A B)=\delta(A) B+A \delta(B)$ for each $A, B$ in $\mathcal{R}$, and $\delta$ is called a Jordan derivation if $\delta\left(A^{2}\right)=\delta(A) A+A \delta(A)$ for every $A$ in $\mathcal{R}$. Obviously, every derivation is a Jordan derivation. The converse is, in general, not true. A classi-

Copyright 2016 by the Tusi Mathematical Research Group.

Received Apr. 1, 2015; Accepted Aug. 18, 2015.

${ }^{*}$ Corresponding author.

2010 Mathematics Subject Classification. Primary 47B47; Secondary 47L35, 47C15.

Keywords. $C^{*}$-algebra, Jordan left derivation, left derivable point, left separating point. 


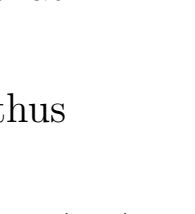

ANALYSIS
Banach J. Math. Anal. 10 (2016), no. 3, 466-481

http://dx.doi.org/10.1215/17358787-3599675

ISSN: $1735-8787$ (electronic)

http://projecteuclid.org/bjma

\title{
CHARACTERIZATIONS OF JORDAN LEFT DERIVATIONS ON SOME ALGEBRAS
}

\author{
GUANGYU AN, YANA DING, and JIANKUI LI* \\ Communicated by A. R. Villena
}

\begin{abstract}
A linear mapping $\delta$ from an algebra $\mathcal{A}$ into a left $\mathcal{A}$-module $\mathcal{M}$ is called a Jordan left derivation if $\delta\left(A^{2}\right)=2 A \delta(A)$ for every $A \in \mathcal{A}$. We prove that if an algebra $\mathcal{A}$ and a left $\mathcal{A}$-module $\mathcal{M}$ satisfy one of the following conditions-(1) $\mathcal{A}$ is a $C^{*}$-algebra and $\mathcal{M}$ is a Banach left $\mathcal{A}$-module; (2) $\mathcal{A}=$ $\operatorname{Alg} \mathcal{L}$ with $\cap\left\{L_{-}: L \in \mathcal{J}_{\mathcal{L}}\right\}=(0)$ and $\mathcal{M}=B(X)$; and (3) $\mathcal{A}$ is a commutative subspace lattice algebra of a von Neumann algebra $\mathcal{B}$ and $\mathcal{M}=B(\mathcal{H})$ - then every Jordan left derivation from $\mathcal{A}$ into $\mathcal{M}$ is zero. $\delta$ is called left derivable at $G \in \mathcal{A}$ if $\delta(A B)=A \delta(B)+B \delta(A)$ for each $A, B \in \mathcal{A}$ with $A B=G$. We show that if $\mathcal{A}$ is a factor von Neumann algebra, $G$ is a left separating point of $\mathcal{A}$ or a nonzero self-adjoint element in $\mathcal{A}$, and $\delta$ is left derivable at $G$, then $\delta \equiv 0$.
\end{abstract}

\section{INTRODUCTION}

Let $\mathcal{R}$ be an associative ring. For an integer $n \geqslant 2, \mathcal{R}$ is said to be $n$-torsion-free if $n A=0$ implies $A=0$ for every $A$ in $\mathcal{R}$. Recall that a ring $\mathcal{R}$ is prime if $A \mathcal{R} B=(0)$ implies that either $A=0$ or $B=0$ for each $A, B$ in $\mathcal{R}$, and it is semiprime if $A \mathcal{R} A=(0)$ implies $A=0$ for every $A$ in $\mathcal{R}$.

Suppose that $\mathcal{M}$ is an $\mathcal{R}$-bimodule. An additive mapping $\delta$ from $\mathcal{R}$ into $\mathcal{M}$ is called a derivation if $\delta(A B)=\delta(A) B+A \delta(B)$ for each $A, B$ in $\mathcal{R}$, and $\delta$ is called a Jordan derivation if $\delta\left(A^{2}\right)=\delta(A) A+A \delta(A)$ for every $A$ in $\mathcal{R}$. Obviously, every derivation is a Jordan derivation. The converse is, in general, not true. A classi-

Copyright 2016 by the Tusi Mathematical Research Group.

Received Apr. 1, 2015; Accepted Aug. 18, 2015.

${ }^{*}$ Corresponding author.

2010 Mathematics Subject Classification. Primary 47B47; Secondary 47L35, 47C15.

Keywords. $C^{*}$-algebra, Jordan left derivation, left derivable point, left separating point. 


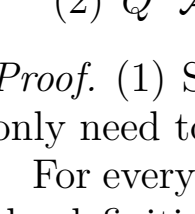

ANALYSIS
Banach J. Math. Anal. 10 (2016), no. 3, 466-481

http://dx.doi.org/10.1215/17358787-3599675

ISSN: $1735-8787$ (electronic)

http://projecteuclid.org/bjma

\title{
CHARACTERIZATIONS OF JORDAN LEFT DERIVATIONS ON SOME ALGEBRAS
}

\author{
GUANGYU AN, YANA DING, and JIANKUI LI* \\ Communicated by A. R. Villena
}

\begin{abstract}
A linear mapping $\delta$ from an algebra $\mathcal{A}$ into a left $\mathcal{A}$-module $\mathcal{M}$ is called a Jordan left derivation if $\delta\left(A^{2}\right)=2 A \delta(A)$ for every $A \in \mathcal{A}$. We prove that if an algebra $\mathcal{A}$ and a left $\mathcal{A}$-module $\mathcal{M}$ satisfy one of the following conditions-(1) $\mathcal{A}$ is a $C^{*}$-algebra and $\mathcal{M}$ is a Banach left $\mathcal{A}$-module; (2) $\mathcal{A}=$ $\operatorname{Alg} \mathcal{L}$ with $\cap\left\{L_{-}: L \in \mathcal{J}_{\mathcal{L}}\right\}=(0)$ and $\mathcal{M}=B(X)$; and (3) $\mathcal{A}$ is a commutative subspace lattice algebra of a von Neumann algebra $\mathcal{B}$ and $\mathcal{M}=B(\mathcal{H})$ - then every Jordan left derivation from $\mathcal{A}$ into $\mathcal{M}$ is zero. $\delta$ is called left derivable at $G \in \mathcal{A}$ if $\delta(A B)=A \delta(B)+B \delta(A)$ for each $A, B \in \mathcal{A}$ with $A B=G$. We show that if $\mathcal{A}$ is a factor von Neumann algebra, $G$ is a left separating point of $\mathcal{A}$ or a nonzero self-adjoint element in $\mathcal{A}$, and $\delta$ is left derivable at $G$, then $\delta \equiv 0$.
\end{abstract}

\section{INTRODUCTION}

Let $\mathcal{R}$ be an associative ring. For an integer $n \geqslant 2, \mathcal{R}$ is said to be $n$-torsion-free if $n A=0$ implies $A=0$ for every $A$ in $\mathcal{R}$. Recall that a ring $\mathcal{R}$ is prime if $A \mathcal{R} B=(0)$ implies that either $A=0$ or $B=0$ for each $A, B$ in $\mathcal{R}$, and it is semiprime if $A \mathcal{R} A=(0)$ implies $A=0$ for every $A$ in $\mathcal{R}$.

Suppose that $\mathcal{M}$ is an $\mathcal{R}$-bimodule. An additive mapping $\delta$ from $\mathcal{R}$ into $\mathcal{M}$ is called a derivation if $\delta(A B)=\delta(A) B+A \delta(B)$ for each $A, B$ in $\mathcal{R}$, and $\delta$ is called a Jordan derivation if $\delta\left(A^{2}\right)=\delta(A) A+A \delta(A)$ for every $A$ in $\mathcal{R}$. Obviously, every derivation is a Jordan derivation. The converse is, in general, not true. A classi-

Copyright 2016 by the Tusi Mathematical Research Group.

Received Apr. 1, 2015; Accepted Aug. 18, 2015.

${ }^{*}$ Corresponding author.

2010 Mathematics Subject Classification. Primary 47B47; Secondary 47L35, 47C15.

Keywords. $C^{*}$-algebra, Jordan left derivation, left derivable point, left separating point. 


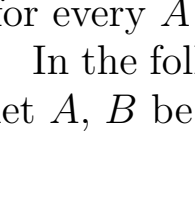

ANALYSIS
Banach J. Math. Anal. 10 (2016), no. 3, 466-481

http://dx.doi.org/10.1215/17358787-3599675

ISSN: $1735-8787$ (electronic)

http://projecteuclid.org/bjma

\title{
CHARACTERIZATIONS OF JORDAN LEFT DERIVATIONS ON SOME ALGEBRAS
}

\author{
GUANGYU AN, YANA DING, and JIANKUI LI* \\ Communicated by A. R. Villena
}

\begin{abstract}
A linear mapping $\delta$ from an algebra $\mathcal{A}$ into a left $\mathcal{A}$-module $\mathcal{M}$ is called a Jordan left derivation if $\delta\left(A^{2}\right)=2 A \delta(A)$ for every $A \in \mathcal{A}$. We prove that if an algebra $\mathcal{A}$ and a left $\mathcal{A}$-module $\mathcal{M}$ satisfy one of the following conditions-(1) $\mathcal{A}$ is a $C^{*}$-algebra and $\mathcal{M}$ is a Banach left $\mathcal{A}$-module; (2) $\mathcal{A}=$ $\operatorname{Alg} \mathcal{L}$ with $\cap\left\{L_{-}: L \in \mathcal{J}_{\mathcal{L}}\right\}=(0)$ and $\mathcal{M}=B(X)$; and (3) $\mathcal{A}$ is a commutative subspace lattice algebra of a von Neumann algebra $\mathcal{B}$ and $\mathcal{M}=B(\mathcal{H})$ - then every Jordan left derivation from $\mathcal{A}$ into $\mathcal{M}$ is zero. $\delta$ is called left derivable at $G \in \mathcal{A}$ if $\delta(A B)=A \delta(B)+B \delta(A)$ for each $A, B \in \mathcal{A}$ with $A B=G$. We show that if $\mathcal{A}$ is a factor von Neumann algebra, $G$ is a left separating point of $\mathcal{A}$ or a nonzero self-adjoint element in $\mathcal{A}$, and $\delta$ is left derivable at $G$, then $\delta \equiv 0$.
\end{abstract}

\section{INTRODUCTION}

Let $\mathcal{R}$ be an associative ring. For an integer $n \geqslant 2, \mathcal{R}$ is said to be $n$-torsion-free if $n A=0$ implies $A=0$ for every $A$ in $\mathcal{R}$. Recall that a ring $\mathcal{R}$ is prime if $A \mathcal{R} B=(0)$ implies that either $A=0$ or $B=0$ for each $A, B$ in $\mathcal{R}$, and it is semiprime if $A \mathcal{R} A=(0)$ implies $A=0$ for every $A$ in $\mathcal{R}$.

Suppose that $\mathcal{M}$ is an $\mathcal{R}$-bimodule. An additive mapping $\delta$ from $\mathcal{R}$ into $\mathcal{M}$ is called a derivation if $\delta(A B)=\delta(A) B+A \delta(B)$ for each $A, B$ in $\mathcal{R}$, and $\delta$ is called a Jordan derivation if $\delta\left(A^{2}\right)=\delta(A) A+A \delta(A)$ for every $A$ in $\mathcal{R}$. Obviously, every derivation is a Jordan derivation. The converse is, in general, not true. A classi-

Copyright 2016 by the Tusi Mathematical Research Group.

Received Apr. 1, 2015; Accepted Aug. 18, 2015.

${ }^{*}$ Corresponding author.

2010 Mathematics Subject Classification. Primary 47B47; Secondary 47L35, 47C15.

Keywords. $C^{*}$-algebra, Jordan left derivation, left derivable point, left separating point. 


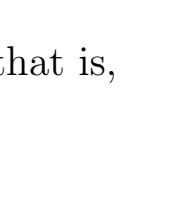

ANALYSIS
Banach J. Math. Anal. 10 (2016), no. 3, 466-481

http://dx.doi.org/10.1215/17358787-3599675

ISSN: $1735-8787$ (electronic)

http://projecteuclid.org/bjma

\title{
CHARACTERIZATIONS OF JORDAN LEFT DERIVATIONS ON SOME ALGEBRAS
}

\author{
GUANGYU AN, YANA DING, and JIANKUI LI* \\ Communicated by A. R. Villena
}

\begin{abstract}
A linear mapping $\delta$ from an algebra $\mathcal{A}$ into a left $\mathcal{A}$-module $\mathcal{M}$ is called a Jordan left derivation if $\delta\left(A^{2}\right)=2 A \delta(A)$ for every $A \in \mathcal{A}$. We prove that if an algebra $\mathcal{A}$ and a left $\mathcal{A}$-module $\mathcal{M}$ satisfy one of the following conditions-(1) $\mathcal{A}$ is a $C^{*}$-algebra and $\mathcal{M}$ is a Banach left $\mathcal{A}$-module; (2) $\mathcal{A}=$ $\operatorname{Alg} \mathcal{L}$ with $\cap\left\{L_{-}: L \in \mathcal{J}_{\mathcal{L}}\right\}=(0)$ and $\mathcal{M}=B(X)$; and (3) $\mathcal{A}$ is a commutative subspace lattice algebra of a von Neumann algebra $\mathcal{B}$ and $\mathcal{M}=B(\mathcal{H})$ - then every Jordan left derivation from $\mathcal{A}$ into $\mathcal{M}$ is zero. $\delta$ is called left derivable at $G \in \mathcal{A}$ if $\delta(A B)=A \delta(B)+B \delta(A)$ for each $A, B \in \mathcal{A}$ with $A B=G$. We show that if $\mathcal{A}$ is a factor von Neumann algebra, $G$ is a left separating point of $\mathcal{A}$ or a nonzero self-adjoint element in $\mathcal{A}$, and $\delta$ is left derivable at $G$, then $\delta \equiv 0$.
\end{abstract}

\section{INTRODUCTION}

Let $\mathcal{R}$ be an associative ring. For an integer $n \geqslant 2, \mathcal{R}$ is said to be $n$-torsion-free if $n A=0$ implies $A=0$ for every $A$ in $\mathcal{R}$. Recall that a ring $\mathcal{R}$ is prime if $A \mathcal{R} B=(0)$ implies that either $A=0$ or $B=0$ for each $A, B$ in $\mathcal{R}$, and it is semiprime if $A \mathcal{R} A=(0)$ implies $A=0$ for every $A$ in $\mathcal{R}$.

Suppose that $\mathcal{M}$ is an $\mathcal{R}$-bimodule. An additive mapping $\delta$ from $\mathcal{R}$ into $\mathcal{M}$ is called a derivation if $\delta(A B)=\delta(A) B+A \delta(B)$ for each $A, B$ in $\mathcal{R}$, and $\delta$ is called a Jordan derivation if $\delta\left(A^{2}\right)=\delta(A) A+A \delta(A)$ for every $A$ in $\mathcal{R}$. Obviously, every derivation is a Jordan derivation. The converse is, in general, not true. A classi-

Copyright 2016 by the Tusi Mathematical Research Group.

Received Apr. 1, 2015; Accepted Aug. 18, 2015.

${ }^{*}$ Corresponding author.

2010 Mathematics Subject Classification. Primary 47B47; Secondary 47L35, 47C15.

Keywords. $C^{*}$-algebra, Jordan left derivation, left derivable point, left separating point. 


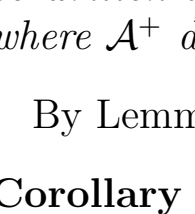

ANALYSIS
Banach J. Math. Anal. 10 (2016), no. 3, 466-481

http://dx.doi.org/10.1215/17358787-3599675

ISSN: $1735-8787$ (electronic)

http://projecteuclid.org/bjma

\title{
CHARACTERIZATIONS OF JORDAN LEFT DERIVATIONS ON SOME ALGEBRAS
}

\author{
GUANGYU AN, YANA DING, and JIANKUI LI* \\ Communicated by A. R. Villena
}

\begin{abstract}
A linear mapping $\delta$ from an algebra $\mathcal{A}$ into a left $\mathcal{A}$-module $\mathcal{M}$ is called a Jordan left derivation if $\delta\left(A^{2}\right)=2 A \delta(A)$ for every $A \in \mathcal{A}$. We prove that if an algebra $\mathcal{A}$ and a left $\mathcal{A}$-module $\mathcal{M}$ satisfy one of the following conditions-(1) $\mathcal{A}$ is a $C^{*}$-algebra and $\mathcal{M}$ is a Banach left $\mathcal{A}$-module; (2) $\mathcal{A}=$ $\operatorname{Alg} \mathcal{L}$ with $\cap\left\{L_{-}: L \in \mathcal{J}_{\mathcal{L}}\right\}=(0)$ and $\mathcal{M}=B(X)$; and (3) $\mathcal{A}$ is a commutative subspace lattice algebra of a von Neumann algebra $\mathcal{B}$ and $\mathcal{M}=B(\mathcal{H})$ - then every Jordan left derivation from $\mathcal{A}$ into $\mathcal{M}$ is zero. $\delta$ is called left derivable at $G \in \mathcal{A}$ if $\delta(A B)=A \delta(B)+B \delta(A)$ for each $A, B \in \mathcal{A}$ with $A B=G$. We show that if $\mathcal{A}$ is a factor von Neumann algebra, $G$ is a left separating point of $\mathcal{A}$ or a nonzero self-adjoint element in $\mathcal{A}$, and $\delta$ is left derivable at $G$, then $\delta \equiv 0$.
\end{abstract}

\section{INTRODUCTION}

Let $\mathcal{R}$ be an associative ring. For an integer $n \geqslant 2, \mathcal{R}$ is said to be $n$-torsion-free if $n A=0$ implies $A=0$ for every $A$ in $\mathcal{R}$. Recall that a ring $\mathcal{R}$ is prime if $A \mathcal{R} B=(0)$ implies that either $A=0$ or $B=0$ for each $A, B$ in $\mathcal{R}$, and it is semiprime if $A \mathcal{R} A=(0)$ implies $A=0$ for every $A$ in $\mathcal{R}$.

Suppose that $\mathcal{M}$ is an $\mathcal{R}$-bimodule. An additive mapping $\delta$ from $\mathcal{R}$ into $\mathcal{M}$ is called a derivation if $\delta(A B)=\delta(A) B+A \delta(B)$ for each $A, B$ in $\mathcal{R}$, and $\delta$ is called a Jordan derivation if $\delta\left(A^{2}\right)=\delta(A) A+A \delta(A)$ for every $A$ in $\mathcal{R}$. Obviously, every derivation is a Jordan derivation. The converse is, in general, not true. A classi-

Copyright 2016 by the Tusi Mathematical Research Group.

Received Apr. 1, 2015; Accepted Aug. 18, 2015.

${ }^{*}$ Corresponding author.

2010 Mathematics Subject Classification. Primary 47B47; Secondary 47L35, 47C15.

Keywords. $C^{*}$-algebra, Jordan left derivation, left derivable point, left separating point. 


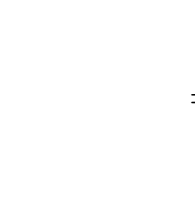

ANALYSIS
Banach J. Math. Anal. 10 (2016), no. 3, 466-481

http://dx.doi.org/10.1215/17358787-3599675

ISSN: $1735-8787$ (electronic)

http://projecteuclid.org/bjma

\title{
CHARACTERIZATIONS OF JORDAN LEFT DERIVATIONS ON SOME ALGEBRAS
}

\author{
GUANGYU AN, YANA DING, and JIANKUI LI* \\ Communicated by A. R. Villena
}

\begin{abstract}
A linear mapping $\delta$ from an algebra $\mathcal{A}$ into a left $\mathcal{A}$-module $\mathcal{M}$ is called a Jordan left derivation if $\delta\left(A^{2}\right)=2 A \delta(A)$ for every $A \in \mathcal{A}$. We prove that if an algebra $\mathcal{A}$ and a left $\mathcal{A}$-module $\mathcal{M}$ satisfy one of the following conditions-(1) $\mathcal{A}$ is a $C^{*}$-algebra and $\mathcal{M}$ is a Banach left $\mathcal{A}$-module; (2) $\mathcal{A}=$ $\operatorname{Alg} \mathcal{L}$ with $\cap\left\{L_{-}: L \in \mathcal{J}_{\mathcal{L}}\right\}=(0)$ and $\mathcal{M}=B(X)$; and (3) $\mathcal{A}$ is a commutative subspace lattice algebra of a von Neumann algebra $\mathcal{B}$ and $\mathcal{M}=B(\mathcal{H})$ - then every Jordan left derivation from $\mathcal{A}$ into $\mathcal{M}$ is zero. $\delta$ is called left derivable at $G \in \mathcal{A}$ if $\delta(A B)=A \delta(B)+B \delta(A)$ for each $A, B \in \mathcal{A}$ with $A B=G$. We show that if $\mathcal{A}$ is a factor von Neumann algebra, $G$ is a left separating point of $\mathcal{A}$ or a nonzero self-adjoint element in $\mathcal{A}$, and $\delta$ is left derivable at $G$, then $\delta \equiv 0$.
\end{abstract}

\section{INTRODUCTION}

Let $\mathcal{R}$ be an associative ring. For an integer $n \geqslant 2, \mathcal{R}$ is said to be $n$-torsion-free if $n A=0$ implies $A=0$ for every $A$ in $\mathcal{R}$. Recall that a ring $\mathcal{R}$ is prime if $A \mathcal{R} B=(0)$ implies that either $A=0$ or $B=0$ for each $A, B$ in $\mathcal{R}$, and it is semiprime if $A \mathcal{R} A=(0)$ implies $A=0$ for every $A$ in $\mathcal{R}$.

Suppose that $\mathcal{M}$ is an $\mathcal{R}$-bimodule. An additive mapping $\delta$ from $\mathcal{R}$ into $\mathcal{M}$ is called a derivation if $\delta(A B)=\delta(A) B+A \delta(B)$ for each $A, B$ in $\mathcal{R}$, and $\delta$ is called a Jordan derivation if $\delta\left(A^{2}\right)=\delta(A) A+A \delta(A)$ for every $A$ in $\mathcal{R}$. Obviously, every derivation is a Jordan derivation. The converse is, in general, not true. A classi-

Copyright 2016 by the Tusi Mathematical Research Group.

Received Apr. 1, 2015; Accepted Aug. 18, 2015.

${ }^{*}$ Corresponding author.

2010 Mathematics Subject Classification. Primary 47B47; Secondary 47L35, 47C15.

Keywords. $C^{*}$-algebra, Jordan left derivation, left derivable point, left separating point. 


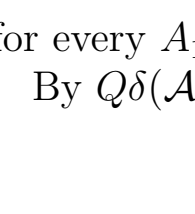

ANALYSIS
Banach J. Math. Anal. 10 (2016), no. 3, 466-481

http://dx.doi.org/10.1215/17358787-3599675

ISSN: $1735-8787$ (electronic)

http://projecteuclid.org/bjma

\title{
CHARACTERIZATIONS OF JORDAN LEFT DERIVATIONS ON SOME ALGEBRAS
}

\author{
GUANGYU AN, YANA DING, and JIANKUI LI* \\ Communicated by A. R. Villena
}

\begin{abstract}
A linear mapping $\delta$ from an algebra $\mathcal{A}$ into a left $\mathcal{A}$-module $\mathcal{M}$ is called a Jordan left derivation if $\delta\left(A^{2}\right)=2 A \delta(A)$ for every $A \in \mathcal{A}$. We prove that if an algebra $\mathcal{A}$ and a left $\mathcal{A}$-module $\mathcal{M}$ satisfy one of the following conditions-(1) $\mathcal{A}$ is a $C^{*}$-algebra and $\mathcal{M}$ is a Banach left $\mathcal{A}$-module; (2) $\mathcal{A}=$ $\operatorname{Alg} \mathcal{L}$ with $\cap\left\{L_{-}: L \in \mathcal{J}_{\mathcal{L}}\right\}=(0)$ and $\mathcal{M}=B(X)$; and (3) $\mathcal{A}$ is a commutative subspace lattice algebra of a von Neumann algebra $\mathcal{B}$ and $\mathcal{M}=B(\mathcal{H})$ - then every Jordan left derivation from $\mathcal{A}$ into $\mathcal{M}$ is zero. $\delta$ is called left derivable at $G \in \mathcal{A}$ if $\delta(A B)=A \delta(B)+B \delta(A)$ for each $A, B \in \mathcal{A}$ with $A B=G$. We show that if $\mathcal{A}$ is a factor von Neumann algebra, $G$ is a left separating point of $\mathcal{A}$ or a nonzero self-adjoint element in $\mathcal{A}$, and $\delta$ is left derivable at $G$, then $\delta \equiv 0$.
\end{abstract}

\section{INTRODUCTION}

Let $\mathcal{R}$ be an associative ring. For an integer $n \geqslant 2, \mathcal{R}$ is said to be $n$-torsion-free if $n A=0$ implies $A=0$ for every $A$ in $\mathcal{R}$. Recall that a ring $\mathcal{R}$ is prime if $A \mathcal{R} B=(0)$ implies that either $A=0$ or $B=0$ for each $A, B$ in $\mathcal{R}$, and it is semiprime if $A \mathcal{R} A=(0)$ implies $A=0$ for every $A$ in $\mathcal{R}$.

Suppose that $\mathcal{M}$ is an $\mathcal{R}$-bimodule. An additive mapping $\delta$ from $\mathcal{R}$ into $\mathcal{M}$ is called a derivation if $\delta(A B)=\delta(A) B+A \delta(B)$ for each $A, B$ in $\mathcal{R}$, and $\delta$ is called a Jordan derivation if $\delta\left(A^{2}\right)=\delta(A) A+A \delta(A)$ for every $A$ in $\mathcal{R}$. Obviously, every derivation is a Jordan derivation. The converse is, in general, not true. A classi-

Copyright 2016 by the Tusi Mathematical Research Group.

Received Apr. 1, 2015; Accepted Aug. 18, 2015.

${ }^{*}$ Corresponding author.

2010 Mathematics Subject Classification. Primary 47B47; Secondary 47L35, 47C15.

Keywords. $C^{*}$-algebra, Jordan left derivation, left derivable point, left separating point. 


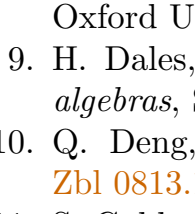

ANALYSIS
Banach J. Math. Anal. 10 (2016), no. 3, 466-481

http://dx.doi.org/10.1215/17358787-3599675

ISSN: $1735-8787$ (electronic)

http://projecteuclid.org/bjma

\title{
CHARACTERIZATIONS OF JORDAN LEFT DERIVATIONS ON SOME ALGEBRAS
}

\author{
GUANGYU AN, YANA DING, and JIANKUI LI* \\ Communicated by A. R. Villena
}

\begin{abstract}
A linear mapping $\delta$ from an algebra $\mathcal{A}$ into a left $\mathcal{A}$-module $\mathcal{M}$ is called a Jordan left derivation if $\delta\left(A^{2}\right)=2 A \delta(A)$ for every $A \in \mathcal{A}$. We prove that if an algebra $\mathcal{A}$ and a left $\mathcal{A}$-module $\mathcal{M}$ satisfy one of the following conditions-(1) $\mathcal{A}$ is a $C^{*}$-algebra and $\mathcal{M}$ is a Banach left $\mathcal{A}$-module; (2) $\mathcal{A}=$ $\operatorname{Alg} \mathcal{L}$ with $\cap\left\{L_{-}: L \in \mathcal{J}_{\mathcal{L}}\right\}=(0)$ and $\mathcal{M}=B(X)$; and (3) $\mathcal{A}$ is a commutative subspace lattice algebra of a von Neumann algebra $\mathcal{B}$ and $\mathcal{M}=B(\mathcal{H})$ - then every Jordan left derivation from $\mathcal{A}$ into $\mathcal{M}$ is zero. $\delta$ is called left derivable at $G \in \mathcal{A}$ if $\delta(A B)=A \delta(B)+B \delta(A)$ for each $A, B \in \mathcal{A}$ with $A B=G$. We show that if $\mathcal{A}$ is a factor von Neumann algebra, $G$ is a left separating point of $\mathcal{A}$ or a nonzero self-adjoint element in $\mathcal{A}$, and $\delta$ is left derivable at $G$, then $\delta \equiv 0$.
\end{abstract}

\section{INTRODUCTION}

Let $\mathcal{R}$ be an associative ring. For an integer $n \geqslant 2, \mathcal{R}$ is said to be $n$-torsion-free if $n A=0$ implies $A=0$ for every $A$ in $\mathcal{R}$. Recall that a ring $\mathcal{R}$ is prime if $A \mathcal{R} B=(0)$ implies that either $A=0$ or $B=0$ for each $A, B$ in $\mathcal{R}$, and it is semiprime if $A \mathcal{R} A=(0)$ implies $A=0$ for every $A$ in $\mathcal{R}$.

Suppose that $\mathcal{M}$ is an $\mathcal{R}$-bimodule. An additive mapping $\delta$ from $\mathcal{R}$ into $\mathcal{M}$ is called a derivation if $\delta(A B)=\delta(A) B+A \delta(B)$ for each $A, B$ in $\mathcal{R}$, and $\delta$ is called a Jordan derivation if $\delta\left(A^{2}\right)=\delta(A) A+A \delta(A)$ for every $A$ in $\mathcal{R}$. Obviously, every derivation is a Jordan derivation. The converse is, in general, not true. A classi-

Copyright 2016 by the Tusi Mathematical Research Group.

Received Apr. 1, 2015; Accepted Aug. 18, 2015.

${ }^{*}$ Corresponding author.

2010 Mathematics Subject Classification. Primary 47B47; Secondary 47L35, 47C15.

Keywords. $C^{*}$-algebra, Jordan left derivation, left derivable point, left separating point. 